\title{
Nondestructive Prediction of Optimal Harvest Time of Cherry Tomatoes Using VIS-NIR Spectroscopy and PLSR Calibration
}

\author{
Haiqing Yang \\ College of Information Engineering, Zhejiang University of Technology, Hangzhou 310032, \\ P.R.China \\ Email: yanghq@zjut.edu.cn
}

\begin{abstract}
Keywords: Tomato; optimal harvest time; visible and near infrared (VIS-NIR) spectroscopy; partial least squares regression (PLSR)
\end{abstract}

\begin{abstract}
In situ determination of optimal harvest time of tomatoes is of value for growers to optimize fruit picking schedule. This study evaluates the feasibility of using visible and near infrared (VIS-NIR) spectroscopy to make an intact estimation of harvest time of tomatoes. A mobile, fibre-type, AgroSpec VIS-NIR spectrophotometer (Tec5, Germany), with a spectral range of $350-2200 \mathrm{~nm}$, was used for spectral acquisition of tomatoes in reflection mode. The harvest time of tomatoes was measured by the days before harvest. After dividing spectra into a calibration set (70\%) and an independent prediction set (30\%), spectra in the calibration set were subjected to a partial least-squares regression (PLSR) with leave-one-out cross validation to establish calibration models. Validation of calibration models on the independent prediction set indicates that the best model can produce excellent prediction accuracy with coefficient of determination $\left(R^{2}\right)$ of 0.90 , root-mean-square error of prediction (RMSEP) of 2.5 days and residual prediction deviation (RPD) of 3.01. It is concluded that VIS-NIR spectroscopy coupled with PLSR models can be adopted successfully for in situ determination of optimal harvest time of tomatoes, which allows for automatic fruit harvest by a horticultural robot.
\end{abstract}

\section{Introduction}

Instant and nondestructive determination of optimal harvest time of tomatoes is significant for tomato growers to implemement fruit harvest automation. However, no standard defination of harvest time of tomatoes has been created. The most widely used methods to predict optimal picking period are based on the physiological patterns of internal properties of tomatoes[1,2]. Nuclear magnetic resonance (NMR) images[3], acoustic impulse-response technique[4], electronic nose[5], quantitative magnetic resonance imaging(MRI) and NMR relaxometry[6] have been tested to measure tomato maturity/ripeness. However, most of these techniques are laboratory-based and cannot be used for in-field fruit measurement.

Recently, visible and near infrared (VIS-NIR) spectroscopy has been widely examined to test internal qualities of off-vine tomato[7-10]. However, there is still lack of information about how to determine harvest time of on-vine tomatoes, which is particularly important for designing a fruit-harvested machine. To our knowledge, no published studies have addressed the use of VIS-NIR spectroscopy for in-field detection of tomatoes.

The objective of this study is to implement the VIS-NIR spectroscopy for in situ determination of optimal harvest time of cherry tomatoes. The paper also aims to evaluate the effect of different spectral transformation on the performance of calibration models.

\section{Materials and Methods}

Tomato Samples. Cherry tomato was cultivated in a greenhouse in the summer growing season 2010. A total of 29 tomatoes were selected from different plants. Measurement started on the $24^{\text {th }}$ July and continued with an interval of 2-3 days until the targeted tomatoes were fully ripe and picked. The statistics of tomatoes were listed in Table 1. 
Table 1 Sample statistics of tomatoes

\begin{tabular}{cccccccc}
\hline \multirow{2}{*}{ Data set } & \multirow{2}{*}{$\begin{array}{c}\text { Fruit } \\
\text { Number }\end{array}$} & \multirow{2}{*}{$\begin{array}{c}\text { Spectral } \\
\text { Number }\end{array}$} & \multicolumn{2}{c}{ Picking Date } & \multicolumn{2}{c}{ Days before harvest } \\
\cline { 5 - 9 } & & & First & Last & Mean & Range & S.D. \\
\hline Cal. & 23 & 217 & 07Aug & 20Aug & 13.85 & $0-27$ & 7.67 \\
Pre. & 6 & 57 & 10Aug & 20Aug & 12.84 & $0-27$ & 7.85 \\
\hline
\end{tabular}

Optical Measurement. The reflectance spectra of tomatoes were measured with a mobile, fibre-type, AgroSpec VIS-NIR spectrophotometer (Tec5, Germany) in reflection mode with spectral region of 350-2200 $\mathrm{nm}$. A USB cable was used for the data transmission between the spectrophotometer and a portable computer. Spectrophotometer scanning was made in three different positions on the equator of a fruit. A total of 10 scans were measured at each position and the spectra from the three positions were averaged as one sample.

Data Analysis and Spectral Transformation. The spectra were divided into a calibration set (70\%) and an independent prediction set $(30 \%)$. The calibration spectra were subjected to a partial leastsquares regression (PLSR) with leave-one-out cross validation, which relates the variations in one response variable (days before harvest) to the variations of several predictors (wavelengths). The optimal number of latent variables (LVs) was determined by minimizing the predicted residual error sum of squares (PRESS).

Before the establishment of PLSR models, it is necessary to eliminate noise, offset and bias in the raw spectra by spectral pretreatments. Due to the low signal-to-noise ratio of both ends of each spectrum, only the region of 400-2100 nm was used for establishing calibration models. Several spectral pretreatment algorithms, including baseline offset correction (BOC), $1^{\text {st }}$ and $2^{\text {nd }}$ detrending and $1^{\text {st }}$ and $2^{\text {nd }}$ derivatives were investigated.

The PLSR models were evaluated using coefficient of determination $\left(R^{2}\right)$, root-mean-square error (RMSE) in calibration and cross validation. The performance of the PLSR models with the independent prediction set was assessed using the coefficient of determination $\left(R^{2}\right)$ and the root-mean-square error of prediction (RMSEP). We also recorded the ratio of residual prediction deviation (RPD), which is the ratio of standard deviation of the measured (reference) data to RMSEP[11,12]. We adopted the criteria of classifying RPD values[23] as follows: an RPD value below 1.5 indicates that the calibration is not usable; an RPD value between 1.5 and 2.0 indicates a possibility to distinguish between high and low values; an RPD value between 2.0 and 2.5 makes approximate quantitative predictions possible. For RPD value between 2.5 and 3.0 and above 3.0, the prediction is classified as good and excellent, respectively. Generally, a good model should have high values of $R^{2}$ and RPD, and low values of RMSEP. The spectral pretreatments and PLSR calibration were conducted using the Unscrambler software (CAMO Software AS, Oslo, Norway).

\section{Results and Discussion}

Spectral Characteristics. A representative reflectance spectra collected from one tomato at different growing stages are shown in Fig. 1. In general, spectral reflectance across the whole range is stronger in less mature tomato and reduces with tomato's ripening. In the visible region, there is a noticeable reflectance valley at around $672 \mathrm{~nm}$, which is linked with the absorption of chlorophyll $a$ in less mature fruit $[14,15]$. Before $12 \mathrm{Aug}$, this reflectance valley gradually decreases down to its minimum. After this, reflectance tends to increase, with dramatic changes during the fully mature stage. However, it does not mean that the real concentration of chlorophyll $a$ reached its maximum on 12 Aug. In fact, the concentration of chlorophyll $a$ in tomato reaches its maximum in the fruit set stage and gradually decreases during its growth[16]. Thus, the measurement of chlorophyll $a$ in tomatoes should be based on more wavelengths rather than at single wavelength at $672 \mathrm{~nm}$. It is worth noting that the reflectance valley (absorbance peak) near $491 \mathrm{~nm}$ decreased with tomato aging and ripening. This band might be linked with lycopene and $\beta$-carotene[10], both synchronized when the tomato turns red. 


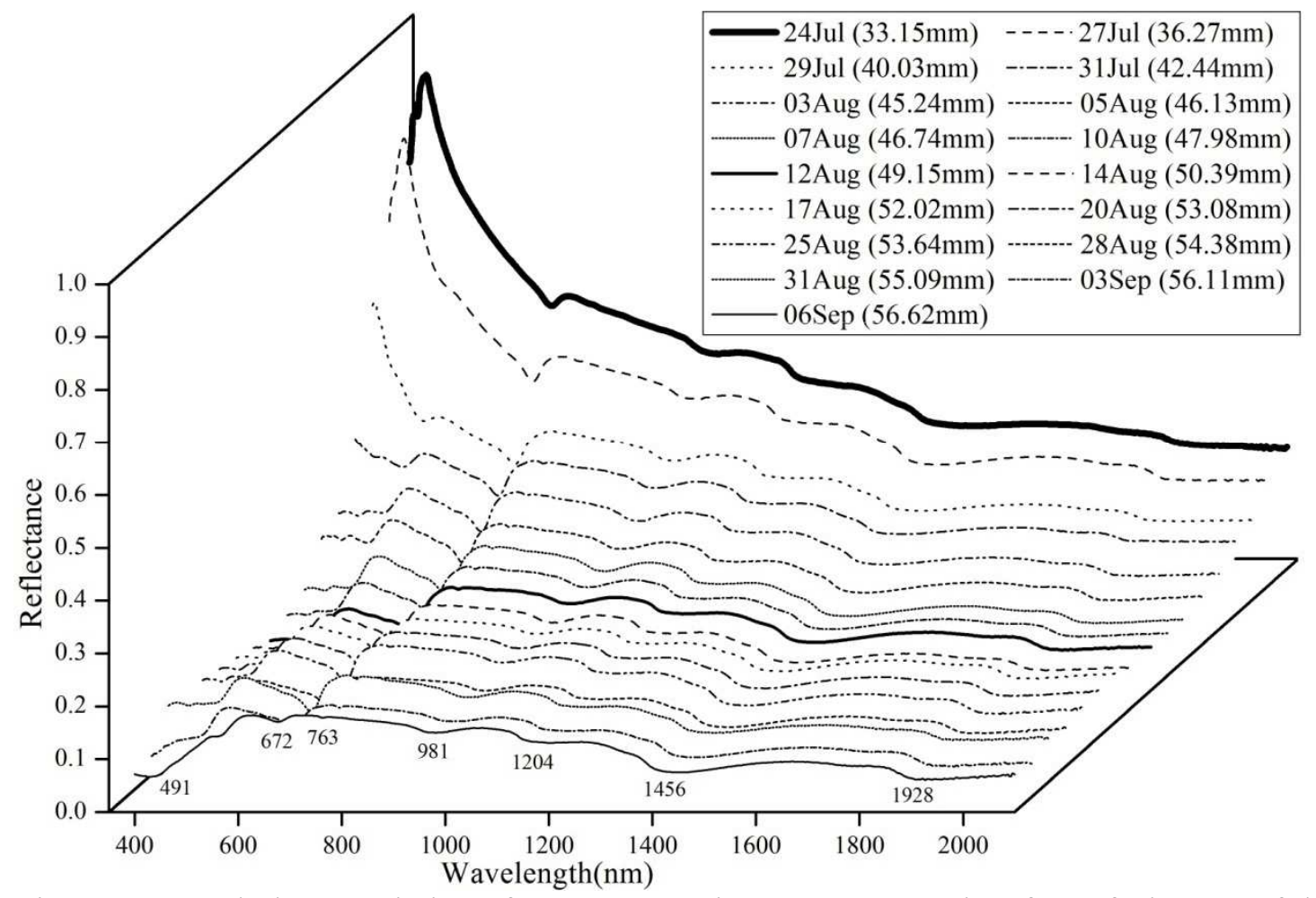

Fig. 1. Spectral characteristics of a representative tomato growing from fruit set to fully ripe. In the parentheses are the changing sizes of the fruit.

In the NIR spectral region, there is a small but noticeable water absorption band at $763 \mathrm{~nm}$, caused by the $3^{\text {rd }}$ overtone of $\mathrm{O}-\mathrm{H}$ stretching[10]. Wide absorption bands of water can be found at $981 \mathrm{~nm}$, corresponding to the $3^{\text {rd }}$ overtone of $\mathrm{H}_{2} \mathrm{O}$ stretching and bending absorbance[10]. Strong water absorption bands are located at around 1456 and $1928 \mathrm{~nm}$, corresponding to the $2^{\text {nd }}$ and $1^{\text {st }}$ overtones of $\mathrm{H}_{2} \mathrm{O}$ stretching and bending, respectively[17]. The band at $1202 \mathrm{~nm}$ can be attributed to sugar absorption. Similar absorption bands were reported in the NIR studies on 'Raf' tomato fruits[9] and nectarines[18].

Prediction Performance of PLSR Models. Table 2 reports the result of the PLSR models for original and transformed spectra. In general, the performance of these models can be regarded as good or excellent, although the accuracy of these PLSR models is a function of spectral pretreatment methods. For instance, the best prediction accuracy was obtained for the spectra transformed by baseline offset correction (BOC) with $R^{2}$ and RPD values of 0.90 and 3.01, respectively.

Table 2 Performance of PLSR models developed for original and tansformed spectra

\begin{tabular}{cccccccccc}
\hline \multirow{2}{*}{ Spectra } & \multirow{2}{*}{ LVs } & \multicolumn{3}{c}{ Calibration } & \multicolumn{3}{c}{ Cross-validation } & \multicolumn{3}{c}{ Independen prediction } \\
\cline { 3 - 9 } & & $R^{2}$ & RMSEC & $R^{2}$ & RMSECV & $R^{2}$ & RMSEP & Bias & RPD \\
\hline Raw & 10 & 0.88 & 2.67 & 0.85 & 3.00 & 0.88 & 2.63 & 0.20 & 2.94 \\
BOC & $\mathbf{1 0}$ & $\mathbf{0 . 8 9}$ & $\mathbf{2 . 5 1}$ & $\mathbf{0 . 8 7}$ & $\mathbf{2 . 8 2}$ & $\mathbf{0 . 9 0}$ & $\mathbf{2 . 5 0}$ & $\mathbf{0 . 3 9}$ & $\mathbf{3 . 0 1}$ \\
$1^{\text {st }}$ Det & 9 & 0.89 & 2.58 & 0.86 & 2.86 & 0.89 & 2.59 & 0.37 & 2.92 \\
$2^{\text {nd }}$ Det & 9 & 0.89 & 2.57 & 0.86 & 2.83 & 0.89 & 2.63 & 1.07 & 2.42 \\
$1^{\text {st }}$ derivative & 7 & 0.88 & 2.65 & 0.82 & 3.26 & 0.88 & 2.71 & 0.37 & 2.79 \\
\hline
\end{tabular}

Note: the units of RMSEC, RMSECV and RMSEP are day. 


\section{Conclusions}

The visible and near infrared (VIS-NIR) spectroscopy was used for in situ spectral acquisition of cherry tomatoes. The harvest time of tomatoes was measured by the days before harvest. A partial least-squares regression (PLSR) was used to build calibration models between the days before harvest and original or transformed spectra. The result shows that the establishment of these PLSR models is successful with prediction coefficient of determination $\left(R^{2}\right)$ of $0.88-0.90$ and residual prediction deviation (RPD) of 2.42-3.01. The minimal prediction error of these models is 2.5 days. It is concluded that VIS-NIR-PLSR method, if coupled with appropriate spectral transformation, can be successfully applied for in situ nondestructive prediction of harvest time of tomatoes, which allows for automatic fruit harvest by a horticultural robot.

\section{Acknowledgements}

The author gratefully thanks the Soil Labs at Cranfield University, UK for the provision of spectrophotometer. Special acknowledgement is given to the Silsoe Horticultural Center, Bedfordshire, UK for supplying tomato plants. This study is financially supported by the Natural Science Foundation of Zhejiang Province, P.R.China (No. Y1090885). The State Scholarship Fund of China also supports the author studying abroad (Grant No.[2009]3004).

\section{References}

[1] A. Peirs, A. Schenk, B.M. Nicolaï, Effect of natural variability among apples on the accuracy of VIS-NIR calibration models for optimal harvest date predictions, Postharvest Biology and Technology 35(2005)1-13.

[2] Y. Makino, M. Ichimura, S. Oshita, Y. Kawagoe, H. Yamanaka, Estimation of oxygen uptake rate of tomato (Lycopersicon esculentum Mill.) fruits by artificial neural networks modeled using near-infrared spectral absorbance and fruit mass, Food Chemistry 121(2010)533-539.

[3] M.E. Saltveit Jr, Determining tomato fruit maturity with nondestructive in vivo nuclear magnetic resonance imaging, Postharvest Biology and Technology 1(1991)153-159.

[4] S. Schotte, N. De Belie, J. De Baerdemaeker, Acoustic impulse-response technique for evaluation and modelling of firmness of tomato fruit, Postharvest Biology and Technology 17 (1999)105-115.

[5] A.H. Gómez, G. Hu, J. Wang, A.G. Pereira, Evaluation of tomato maturity by electronic nose, Computers and Electronics in Agriculture 54(2006)44-52.

[6] M. Musse, S. Quellec, M. Cambert, M.F. Devaux, M. Lahaye, F. Mariette, Monitoring the postharvest ripening of tomato fruit using quantitative MRI and NMR relaxometry, Postharvest Biology and Technology 53(2009)22-35.

[7] A.M.K. Pedro, M.M.C. Ferreira, Simultaneously calibrating solids, sugars and acidity of tomato products using PLS2 and NIR spectroscopy, Analytica Chimica Acta 595(2007)221- 227.

[8] K. Flores, M.T. Sánchez, D. Pérez-Marín, J.E. Guerrero, A. Garrido-Varo, Feasibility in NIRS instruments for predicting internal quality in intact tomato, Journal of Food Engineering 91 (2009)311-318.

[9] R. Choudhary, T.J. Bowser, P. Weckler, N.O. Maness, W. McGlynn, Rapid estimation of lycopene concentration in watermelon and tomato puree by fiber optic visible reflectance spectroscopy, Postharvest Biology and Technology 52(2009)103-109. 
[10] A. Clément, M. Dorais, M. Vernon, Multivariate Approach to the Measurement of Tomato Maturity and Gustatory Attributes and Their Rapid Assessment by Vis-NIR Spectroscopy, Jounal of Agricultural \& Food Chemistry 56(2008)1538-1544.

[11] A. Peirs, J. Lammertyn, K. Ooms, B.M. Nicolaï, Prediction of the optimal picking date of different apple cultivars by means of VIS/NIR-spectroscopy. Postharvest Biology and Technology 21(2001)189-199.

[12] P.C. Williams, Variables affecting near-infrared reflectance spectroscopic analysis, in: P.C. Williams, K.H. Norris (eds.) AACC, St. Paul, MN, 1987, pp.143-166.

[13] A.M. Mouazen, J.D. Baerdemaeker, H. Ramon, Effect of wavelength range on the measurement accuracy of some selected soil constituents using visual-near infrared spectroscopy, Journal of Near Infrared Spectroscopy 14(2006)189-199.

[14] L.M.M. Tijskens, P.E. Zerbini, R.E. Schouten, M. Vanoli, S. Jacob, M. Grassi, R. Cubeddu, L. Spinelli, A. Torricelli, Assessing harvest maturity in nectarines, Postharvest Biology and Technology 45(2007)204-213.

[15] V. Ziosi, M. Noferini, G. Fiori, A. Tadiello, L. Trainotti, G. Casadoro, G. Costa, A new index based on vis spectroscopy to characterize the progression of ripening in peach fruit, Postharvest Biology and Technology 49(2008)319-329.

[16] S.H. Choi, S.H. Lee, H.J. Kim, I.S. Lee, N. Kozukue, C.E. Levin, M. Friedman, Changes in Free Amino Acid, Phenolic, Chlorophyll, Carotenoid, and Glycoalkaloid Contents in Tomatoes during 11 Stages of Growth and Inhibition of Cervical and Lung Human Cancer Cells by Green Tomato Extracts, Journal of Agricultural and Food Chemistry 58(2010), 7547- 7556.

[17] I. Murry, P.C. Williams, Chemical Principals of Near-infrared technology, in: P.C. Williams, K.H. Norris (eds.) AACC, St. Paul, MN, 1987, pp.17-34.

[18] D. Pérez-Marín, M.T. Sánchez, P. Paz, M.A. Soriano, J.E. Guerrero, A. Garrido-Varo, Non-destructive determination of quality parameters in nectarines during on-tree ripening and postharvest storage, Postharvest Biology and Technology 52(2009)180-188. 